not result in onward transmission. In addition recent changes in the national guidelines for providing post exposure prophylaxis with immunoglobulin to young infants presented logistical challenges when delivered in the hospital setting.

\section{GP204 HIV INFECTION IN CHILDREN WITH PERINATAL TRANSMISSION: CLINICAL AND IMMUNOLOGICAL FEATURES}

\begin{abstract}
'Ekaterina Moskaleva, 'Alla Petrova, ${ }^{2}$ Svetlana Smirova, 'Lyubov Rychkova, ${ }^{1}$ Anastasiya Vanyarkina*. 'Scientific Centre for Family Health and Human Reproduction Problems, Irkutsk, Russian Federation; ${ }^{2}$ Scientific Research Institute of Medical Problems of the North, Krasnoyarsk, Russian Federation
\end{abstract}

10.1136/archdischild-2019-epa.263

Introduction HIV infection is more rapid and serious in children with perinatal transmission. As the immune system is progressively deteriorating, the aim was to study parameters of the immune system, which is necessary to determine disease approach and prognosis.

Methods We conducted a cross-sectional randomized study of children aged 1 to 5 years: 32 children with perinatal HIV infection (main group), and 28 healthy children. Criteria for inclusion in main group: confirmed HIV infection, clinical stages 2-3 (WHO classification, 2006). Exclusion criterion is parents' refusal to participate. Clinical examination of children was conducted. We estimated the frequency of clinical manifestations of immunodeficiency syndromes $\left(\mathrm{P}_{\mathrm{abs}} / \mathrm{P} \%\right)$, and analyzed parameters of the immune status. The pattern of lymphocyte subpopulations was assessed by laser flow cytometry, immunoglobulins and interleukins concentrations were determined by EIA. A comparative analysis was performed using non-parametric Mann-Whitney U-test.

Results Regardless of clinical stage, all children with HIV infection showed delay in physical and neuropsychic development, CNS damage, Banti's syndrome, bulky lymphadenopathy, recurrent bacterial infections of upper respiratory tract. Frequent manifestations of HIV infection are anemia of 90.5 $\pm 3.7 \%$, and repeated pneumonia from 2 to 6 times a year $(71.4 \pm 5.7 \%)$. Immunity disorders manifested themselves in all HIV-infected children. Infectious and lymphoproliferative syndromes have the most intensive manifestation and are found in $100 \%$ of the HIV-infected. Infections target organs and systems, functioning as a barrier and participating in immunogenesis.

Combined damage to the immune system was detected. The state of cellular immunity was characterized by decreased numbers of CD3 + lymphocytes $(55.3 \pm 7.05 \%$; $\mathrm{p}<0.01)$, CD $4+$ lymphocytes $(26.15 \pm 5.6 \% ; \mathrm{p}<0.001)$, and increased absolute $(0.07 \pm 0.01 ; \mathrm{p}<0.01)$ and relative $(1.7$ $\left.\pm 0.9 \times 10^{9} / 1 ; \mathrm{p}<0.001\right)$ numbers of $\mathrm{NK}$ cells. Changes in humoral immune response were expressed by a significant increase in concentration of serum IgA $(1.14 \pm 0.51 \mathrm{~g} / \mathrm{l}), \mathrm{IgM}$ $(2.59 \pm 1.51 \mathrm{~g} / \mathrm{l}, \mathrm{IgG}(22.17 \pm 2.2 \mathrm{~g} / \mathrm{l})$, and circulating immune complexes by $2-4$ times in comparison with the healthy children. Study of serum concentrations of INF- $\gamma$, IL-4, IL-1, IL8 in children with HIV infection revealed a significant increase in INF- $\gamma$, and a decrease in IL-4, IL-1, IL-8 in comparison with healthy group.

Conclusions We revealed signs of the immune system impairment in children with perinatal HIV infection: clinical - manifestations of infectious and lymphoproliferative syndromes, and laboratory - combined immunodeficiency. HIV-infected children had an immune deviation towards Th2 response: increased IL-4 concentration and total IgE, and decreased INF- $\gamma$ in serum.

\section{GP205 MATERNAL PYREXIA AT BIRTH; A TRUE NEONATAL INFECTION OR NOT?}

Muhammad Abu Bakar, Aisha ljaz*, Aftab, Niazy Al-Assaf. University maternity hospital limerick, Limerick, Ireland

\subsection{6/archdischild-2019-epa.264}

Background Early onset neonatal sepsis(EOS) is defined as infection in the first 72 hours of life ${ }^{1}$. It can cause serious morbidity and mortality therefore prophylactic antibiotics are required for high risk infants. ${ }^{1}$

Objective To determine the rate of positive blood cultures in babies treated in view of maternal pyrexia. To compare maternal high CRP and blood cultures with babies CRP and blood cultures.

Standard NICE guidelines Neonatal infection (early onset): antibiotics for prevention and treatment published: 22 August 2012. ${ }^{2}$

Methods Retrospective chart review was done on term babies admitted to NICU with maternal pyrexia form July 2016 to December 2016. Details of babies blood culture, CRP, and further workup e.g CSF studies and PCR studies were collected. Other details of maternal septic workup were also recorded. Data was compiled on Microsoft excel sheet and results analyzed.

Results Total 52 babies admitted from July 2016 to December 2016 in NICU with suspected EOS. 31\%(n=16) have other risk factors for EOS like maternal GBS, chorioamnionitis or PROM were excluded from the study. Only $3 \%(n=1)$ blood culture came back Micrococcus Luteus positive(contamination) with negative maternal culture. None of the mother involved in this study had positive blood culture. $28 \%(n=10)$ of the babies had CRP in the range of 6 to $30 \mathrm{mg} / \mathrm{L}$ while $3 \%(\mathrm{n}=1)$ of the babies had CRP above $50 \mathrm{mg} / \mathrm{L} .59 \%(\mathrm{n}=20)$ of the maternal CRP were less than $50 \mathrm{mg} / \mathrm{L}$. Only $6 \%(\mathrm{n}=2)$ of the mothers had CRP more than $200 \mathrm{mg} / \mathrm{L}$ compared to babies CRP less than $5 \mathrm{mg} / \mathrm{L}$.

Conclusion No correlation between baby CRP and maternal CRP in view of maternal pyrexia. None of the babies had positive blood culture in this cohort. Treating babies in view of maternal pyrexia alone as the risk factor for EOS should be reviewed to restrict unnecessary antibiotics use.

\section{REFERENCES}

1. Emily J. Weston, MPH, Tracy Pondo, MSPH, Melissa M. Lewis, MPH, Pat MartellCleary, MSW: The Burden of Invasive Early-Onset Neonatal Sepsis in the United States 2005-2008 Pediatr Infect Dis J. 2011 Nov; 30(11): 937-941

2. NICE guidelines: Neonatal infection(early onset): antibiotics for prevention and treatment(CG149); August 2012 